\title{
Crystal analyzers for indirect-geometry broadband neutron spectrometers: adding reality to idealized design
}

\author{
M. Zanetti, F. Masi, S. Rudic, J. Armstrong, S.F. Parker, \\ F. Fernandez-Alonso and G. Gorini
}

\section{Published version information}

Citation: M Zanetti et al. 'Crystal analyzers for indirect-geometry broadband neutron spectrometers: adding reality to idealized design.' Journal of Surface Investigation: X-ray, Synchrotron and Neutron Techniques, vol. 14, no. S1 (2020): S242-S250.

\section{DOI: $\underline{10.1134 / S 1027451020070526}$}

This is a post-peer-review, pre-copyedit version of an article published in the Journal of Surface Investigation: X-ray, Synchrotron and Neutron Techniques. The final authenticated version is available online at the DOI above.

This version is made available in accordance with publisher policies. Please cite only the published version using the reference above. This is the citation assigned by the publisher at the time of issuing the AAM. Please check the publisher's website for any updates. 


\title{
Crystal Analysers for Indirect-Geometry Broadband Neutron Spectrometers: Adding Reality to Idealised Design
}

\author{
M. Zanetti ${ }^{\mathrm{a}, \mathrm{b}, *}$, F. Masi ${ }^{\mathrm{b}}$, S. Rudić ch,* J. Armstrong $^{\mathrm{b}}$, S.F. Parker ${ }^{\mathrm{b}}$, F. \\ Fernandez-Alonso ${ }^{\mathrm{f}, \mathrm{g}, \mathrm{c}}, \mathrm{G}$. Gorini ${ }^{\mathrm{d}, \mathrm{e}}$ \\ ${ }^{a}$ Consiglio Nazionale delle Ricerche, Dipartimento di Scienze Fisiche e Tecnologie della Materia, \\ Roma, Italia \\ ${ }^{b}$ ISIS Facility, Rutherford Appleton Laboratory, Chilton, Didcot, Oxfordshire OX11 0QX, United \\ Kingdom \\ ${ }^{c}$ Department of Physics and Astronomy, University College London, Gower Street, London \\ WC1E 6BT, United Kingdom \\ ${ }^{d}$ Università degli Studi di Milano-Bicocca, Piazza della Scienza 3, 20126 Milano, Italia \\ "Istituto di Fisica del Plasma "P. Caldirola", CNR-IFP, Milano, Italia \\ ${ }^{f}$ Materials Physics Center, CSIC-UPV/EHU, Paseo Manuel Lardizabal 5, 20018 Donostia - San \\ Sebastian, Spain \\ ${ }^{g}$ IKERBASQUE, Basque Foundation for Science, Maria Diaz de Haro 3, 48013 Bilbao, Spain
}

\begin{abstract}
The recent trend for indirect-geometry broadband neutron spectrometers to fully exploit available neutrons at pulsed spallation sources, seeks to improve their performance via the use of large-area curved analysers, which focus neutrons in time, energy and space. Herein, we describe ongoing, joint efforts between CNR (IT) and ISIS (UK) aimed at future upgrades of TOSCA at ISIS, that are also essential for the design, and subsequent construction, of VESPA at the ESS. Such an improvement in performance arises from both a larger detector coverage using a curved geometry, as well as appropriate tuning of analyser-crystal characteristics. To achieve high resolution over the entire spectral range of $0-500 \mathrm{meV}$, timefocusing of neutrons from the sample to the detector must be achieved within tight tolerances. The effect of graphite mosaicity and its variation from crystalto-crystal across the whole analyser, as well as the impact of manufacturing tol-
\end{abstract}

\footnotetext{
${ }^{*}$ Corresponding author

Email addresses: matteo.zanetti@amministrazione.cnr.it (M. Zanetti ), svemir.rudic@stfc.ac.uk (S. Rudić )
} 
erances on instrument performance, and of the detector pixelation are here investigated so as to inform the design, with a view to balancing neutron collection performance against resolution requirements.

Keywords: indirect geometry neutron instrument, neutron spectrometer, energy analyser, TOSCA, VESPA

\section{Introduction}

Indirect-geometry Time-of-Flight (ToF) broadband $\left(-20\right.$ to $\left.4000 \mathrm{~cm}^{-1}\right)$ neutron spectrometers rely on the selection of inelastically scattered neutrons with a set neutron energy. The most efficient approach to achieve this goal, as demonstrated by 20 years of TOSCA operations [1-5], is by collecting neutrons via High Oriented Pyrolytic Graphite (HOPG) analysers placed at about $45^{\circ}$ and $135^{\circ}$ scattering angles, on a plane parallel to the sample axis. The analysers select the neutrons through the $\left(\begin{array}{lll}0 & 0 & 2\end{array}\right)$ reflection, and direct them to detectors placed as near as possible to the sample plane. Beryllium filters downstream from the analysers provide the suppression of higher-order reflections. This kind of configuration provides the so-called time focusing, which allows all selected neutrons to travel to the detectors within the same time-frame, and practically cancelling the uncertainty on the ToF after scattering from the sample. This feature is fundamental to provide high resolution at high energy transfers (above hundreds of $\mathrm{cm}^{-1}$ ).

In an effort to fully exploit available neutrons at pulsed spallation sources, the use of curved analysers able to focus neutrons in time, energy and space is envisaged to increase performance, by collecting scattered neutrons over large solid angles as implemented on VISION [6] at SNS, overcoming the space constraints inherent with other geometries. The adoption of high-mosaic HOPG can also contribute to enhancing the neutron collection [7], by increasing the analyser angular acceptance. The analysers need to be built from small HOPG tiles that allow for a multifaceted curved surface. In principle, the adoption of a curved analyser does not guarantee time focusing, however, this may be obtained to a certain extent (i.e. limiting the uncertainty to a certain threshold) by tuning the position and the orientation of each of the HOPG tiles [8]. Energy and, most importantly, time focussing need to be provided by the analysers.

Here, we provide insights into the design of this type of analyser, starting from the most ideal conditions and then evaluating the effects of the uncertainties in their physical realization. These analyses have been performed as part of the design of two instruments: the VESPA [8] spectrometer, jointly developed by 
the Italian "Consiglio Nazionale delle Ricerche" (CNR) and the "ISIS Neutron and Muon Source" (UK) for the "European Spallation Source" (Sweden); and the upgrade of the TOSCA spectrometer (ISIS, UK). In particular, the results here reported are based on the VESPA layout [8, 9], in its High-Resolution mode, but the same conclusions are applicable to TOSCA. The assessment of the reported results is being performed on TOSCA, using ad hoc test rigs.

\section{Theoretical Analysis: Ideal Design}

When designing the analysers to cover large solid angles, generally one has to rely on simple, ideal, mathematical principles in order to define a curvature that allows collecting the highest possible number of scattered neutrons without deteriorating the resolution beyond a given threshold. The resolution of broadband neutron spectrometers has been described in [10, 11]. By taking the most important contributions identified there, and removing the assumption of time focusing, the resolution function can be represented as:

$$
\sigma^{2}(E) \propto \sigma^{2}\left(E_{i}\right)+E_{i}^{2} \frac{\sigma^{2}\left(t_{i}\right)}{t_{i}^{2}}+E_{i}^{2} \frac{\sigma^{2}\left(t_{f}\right)}{\left(t_{i}+t_{f}\right)^{2}}+\sigma^{2}\left(E_{f}\right) .
$$

Where $E$ is the energy transfer, $E_{i}$ is the incident energy, $t_{i}$ is the time of flight from moderator to sample (primary flight path), $t_{f}$ is the ToF from sample to detector and $E_{f}$ is the final energy selected by the analyser. In this work, the resolution is generally stated as a standard deviation $\sigma$, or equivalent FWHM $\Delta E$, which is derived from $\sigma$ assuming a Gaussian shape of the overall instrumental response. This conversion is reliable for high-energy transfers (i.e. $>60 \mathrm{meV}$ ), but overestimates the peak width in the elastic region.

It is evident that the absence of focusing (i.e. $\sigma^{2}\left(t_{f}\right)$ not equal to zero) leads to a deterioration of resolution, as the energy transfer increases. When such an uncertainty arises, it is convolved with the time dispersion from the primary flight path, which in relative terms increases as ToF decreases. The uncertainty effects coming from the primary flight path can be reduced by increasing the path length, but nevertheless, there will be a distance at which the two contributions will match and the effect from the absence of analyser focusing will be dominant, thus setting the limit on the balance between intensity and resolution. The contributions to the resolution can be described with analytical formulae [10, 11] or estimated independently via Monte Carlo simulations, and then summed in quadrature as in Eq.(1). In particular, in our analyses, we used an exact analytic estimation 
of the primary flight path terms and simulated the ones due to the analysers [8], with the McStas [12] package. This approach gives results which are in very good agreement with the ones obtained with a full simulation of a " $\delta$-sample", as described previously [8].

To keep under control the secondary ToF dispersion (STFD) $\sigma^{2}\left(t_{f}\right)$ when large analysers are used, the approach is not based on changes of the instrument size, but on the geometrical focusing of the analyser on a position-sensitive detector array. In this way, all neutrons are forced to travel within the same time to a given detector pixel, which should be reasonably small size as $t_{f}$ and $E_{f}$ can differ over the whole detector bank, and for each of which Eq.(1) can be applied. This can be attained by placing the HOPG tiles over a locus of points that is defined by the following procedure, using Fig. 1 as reference.

[Figure 1 about here.]

The locus is defined in two dimensions, so as to obtain a "vertical" curvature, from which the three-dimensional analyser can be obtained by revolution around the sample axis. With the sample as the origin of a 2-D space and a detector plane shifted with respect to the sample axis (so as to accommodate two detector banks back-to-back), one can select a "starting" point that will be part of the analyser (ideally, the closest acceptable position to the beam) and the desired "starting" $E_{f}$ selected from that position (a "starting" energy). This condition determines the focus point in the detector plane, which will be the same for each position in the 2-D plane and eventually on the analyser. Also, the ToF $t_{\text {set }}$ desired for all the neutrons that should reach the focus point can be determined. Given two fixed points, the reflection plane in any third point of space that allows a neutron to connect them (i.e. travelling from the sample over the vector $\boldsymbol{V}$ and then on the reflected vector $\boldsymbol{R}$ to the focus point) is necessarily defined. By applying Bragg's Law at the reflection plane, the energy (i.e. the velocity) of the neutrons is set and so is the time of flight $t_{f}$ along the path identified by $\boldsymbol{R}$ and $\boldsymbol{V}$. In this way, the whole space can be mapped with respect to $t_{f}$ and $E_{f}$ for each neutron travelling from the sample to the focus point. From this map, the locus of points which satisfies the condition that $t_{f}$ is kept the same for each neutron can be identified. Similarly, it would be possible to identify the locus of constant final energy, a prospect which is more useful for backscattering quasielastic neutron spectrometers [13]. The analyser is then designed by selecting part of the locus so as to cover the desired solid angle within energy constraints, such as those set by the beryllium filter. The following set of equations represents the mathematical interpretation of 
the procedure described (see Fig. 1):

$$
\begin{aligned}
& \boldsymbol{N}=\frac{1}{2}(\boldsymbol{R}-\boldsymbol{V}), \\
& \alpha_{\text {tile }}=\operatorname{atan}\left(\frac{\boldsymbol{N} \cdot \hat{\boldsymbol{y}}}{\boldsymbol{N} \cdot \hat{\boldsymbol{x}}}\right), \\
& \theta=\frac{1}{2}(\operatorname{acos}(\boldsymbol{V} \cdot \boldsymbol{R})), \\
& t=A 2 d \sin (\theta)(|\boldsymbol{R}|+|\boldsymbol{V}|) .
\end{aligned}
$$

In Eq.(5), $\mathrm{A}$ is a conversion factor (constant). The locus is then transformed to a polygonal approximation, in which the HOPG tile dimension is used as the basic unit of length, and the tile orientation is determined by the energy mapping, with respect to their centre, by applying Bragg's Law. Notice that, following this last constraint, the reflection plane for each point of the locus is not necessarily tangent to the curvature that the locus identifies.

If the tiles where infinitely small and with a negligible mosaicity, the uncertainty on the secondary ToF would be zero and all the neutrons would end at the focal point. However, in practice, a small uncertainty arises from these nonidealities and it needs to be kept within a threshold, i.e. $\sigma\left(t_{f}\right)$, by changing the generating parameters of the locus, while the neutrons spread over a larger spot around the focal point. A working assumption is that the smallest tile available from manufacturers will be used, which means a basic unit of length of about $1-1.2 \mathrm{~cm}$.

Once the analyser geometry is set, the mosaic spread of the HOPG crystals used to build it can be optimised. In general, a small mosaicity contributes to better focusing, which entails better resolution. On the other hand, a high mosaicity allows for a larger angular acceptance, and thus an increase in the number of collected neutrons [7]. Once again, collection efficiency and resolution can be matched by carefully saturating the existing resolution before a detriment becomes apparent. Furthermore, the production, and the costs associated with large quantities of tiles to cover large areas, are eased as the mosaicity increases. It is worth noting that high-mosaicity $\left(\right.$ i.e. $>3.5^{\circ}$ ) HOPG is not readily available, and further developments might be required. 


\section{Method: Uncertainties and their simulation}

The design of the analysers, as carried out up to this point, is based on three assumptions: (I) the HOPG tiles are exactly placed and aligned on the desired curvature; (II) the mosaicity value is constant for each tile; and (III) the detectors lie on an ideal plane.

Given the manufacturing tolerances for the different analyser components, concerns can be raised on the consistency of the analyser construction. In order to evaluate this effect, we investigated points (I) and (II) via analog Monte Carlo (MC) simulations, in which the random variable is an analyser configuration. First, random displacements and rotations, sampled uniformly within the manufacturing tolerance ranges, have been assigned independently to each tile of the VESPA analyser, while keeping the mosaicity level constant. For the second assumption, a random mosaicity value, sampled uniformly between crystal tolerances, has been assigned to each tile of the VESPA analyser, while keeping the tile positions fixed to the ideal ones. In both cases, HOPG has been modelled as an ideal monochromator, neglecting thickness effects. These procedures have been repeated 300 times, a typical sample size for a large population, which provides a 94\% confidence level, in order to estimate the variations of resolution and intensity, for different tolerance ranges on either manufacturing tolerances or HOPG mosaic spread. Given the nature of a MC simulation, there is an uncertainty associated with the $\sigma\left(t_{f}\right)$ and $\sigma\left(E_{f}\right)$, or an "uncertainty of the uncertainty", UoU.

Finally, the path that a neutron travels into the sensitive volume of a detector can further affect STFD. On TOSCA, squashed ${ }^{3} \mathrm{He}$ tubes (the nearest option to an ideal "flat" geometry) are used, however, this kind of detector is not anymore available, and it does not provide position sensitivity. Thus, we tested the effect of detector voxel choice with realistic dimensions, and also considering the ${ }^{3} \mathrm{He}$ tube geometry. In order to evaluate the effect, we added a random time to the final ToF during the simulations, performing a sampling from the time of travel through the detector for each simulated neutron. The random time is selected from a uniform distribution, assuming $100 \%$ detector efficiency for each of the available $E_{f}$, as it would be for a high-pressure ${ }^{3} \mathrm{He}$ detector tube. For lower efficiencies, the distribution is smoothed and peaked at the detector centreline, so this assumption is a worst-case scenario from the point of view of voxel size, in particular for the fastest desirable neutrons $(\sim 5 \mathrm{meV})$. We considered initially position-sensitive (PS) assemblies of box voxels, with different sizes and volumes, to establish the best configuration of a speculative high-PS-resolution detector; and then two realistic tube sizes for comparison to the ideal, flat geometry. 


\section{Results and Discussion}

Here, we present the use of the design procedure described above: mosaicspread optimization and tolerance effects.

An example of the results obtained by adopting the design procedure [Eq. (2,5)] is reported in Fig. 2 for a "starting" energy of $4 \mathrm{meV}$, a starting point $(0.1615 \mathrm{~m}, 0.065 \mathrm{~m})$, and a detector plane $\mathrm{x}=0.025 \mathrm{~m}$. This set of generating conditions was used for the VESPA layout. In principle, higher energies (i.e. shorter ToFs) are achieved with curvatures closer to the sample axis. The final curvature, identified with a figure of merit, defined as $\ln \left[\left(t-t_{\text {set }}\right)^{2}\right]$, is an ellipse, but the sample and the focus point do not lie at the ellipse focus, since the tiles are not placed tangentially to the ellipse, but are oriented according to Eq. 3 .

To assess the effects of the HOPG mosaicity on neutron collection, a parameter sweep has been performed for this layout, ranging from $1^{\circ}$ to $6^{\circ}$ with increments of half a degree. There is a nearly linear gain in the intensity delivered to the detector bank (Fig. 3 3 ). The focusing spot, defined as the detector area in which the intensity is within 2 orders of magnitude with respect to the maximum, increases as well (Fig. $3[\mathrm{~b}-\mathrm{c}$ ), thus the local count rate is not dramatically increased and the detector area is better used. Concerning the main resolution parameters, which are the $\sigma\left(E_{f}\right)$ and $\sigma\left(t_{f}\right)$, both increase (Fig. 4), linearly at least to $4^{\circ}$. The thresholds on these parameters can be set by considering the desired final resolution of the instrument, including all the contributions from Eq. 11. For instance, for VESPA in High-Resolution mode, assuming a $4^{\circ}$ mosaicity, the detriment at $500 \mathrm{meV}$ is not excessive, being a $1.2 \%$ relative resolution, while in the fingerprint region the resolution is always below 1\%, as reported in Fig. 5, which shows how the spectral resolution across the energy-transfer range of the instrument deteriorates as the mosaicity is increased.

[Figure 2 about here.]

[Figure 3 about here.]

[Figure 4 about here.]

[Figure 5 about here.]

When mechanical tolerances are considered (see Fig. 6 for the reference system), it is shown that reasonable and achievable tolerances (max $0.2 \mathrm{~mm}$ translation; and $\max 0.2^{\circ}$ rotation that corresponds to a $0.02 \mathrm{~mm}$ displacement of the 
edge with respect to the tile centre) give results consistent with the ideal case both for resolution and for intensity on the detector bank. The relative variation of the resolution is below $1 \%$ and with a reasonably small relative uncertainty with respect to the average value. The results for different sets of tolerances are reported in Figs. 7f, 8 . Low energy transfers are more sensitive to uncertainties associated with manufacturing tolerances. The different sets of tolerances have been selected heuristically, based on experience of different manufacturing processes. The spread of uncertainties derived from the different configurations tested shows that the centroid of the distributions shifts to larger values for both $t_{f}$ and $E_{f}$, with the uncertainty on the final energy being the most affected. Thus, UoU is larger at lower energy transfers. The distributions of intensity shift towards lower values than ideal and they broaden as well, by a few percent, as the tolerance ranges increase.

[Figure 6 about here.]

[Figure 7 about here.]

[Figure 8 about here.]

A number of mosaic-spread distributions have been considered (commercially available $2^{\circ}-3^{\circ}$, or speculative), in particular with reference to the adoption of high-mosaic HOPG. The mosaicity of each tile can be tested before its use, so it is also possible to obtain tighter tolerances than standard. The results are reported in Figs. 9, 10, It is shown that the relative uncertainty on resolution for standard $2^{\circ}-3^{\circ}$ HOPG is on average $0.3 \%$, a perfectly acceptable value, and that the intensity varies within a few percent with respect to the nominal case (i.e. uniform $2.5^{\circ}$ over the whole analyser). Concerning the tolerance range for a hypothetical $4^{\circ}$ mosaic HOPG, a tolerance of $\pm 0.5^{\circ}$ gives a relative performance similar to the one obtained with standard HOPG. The spread of uncertainties derived from the different configurations tested shows that the ToF uncertainty is affected by a centroid shift to larger values, but the uncertainty variations of $t_{f}$ and $E_{f}$ are contained with a maximum of $3 \%$ and $1.7 \%$, respectively, for the worst cases. As the footprint of the dispersion increases similarly in both time and energy, the UoU is nearly evenly spread above the spectrometer bandwidth. The distribution of intensity is centred at the ideal value and it broadens, by few percent, as the tolerance increases.

[Figure 9 about here.] 
[Figure 10 about here.]

Studies on detector pixelation include constant voxel volume (Fig. 11| [1), constant pixel area and variable thickness (Fig. 11p) and finally, constant thickness with variable pixel areas and aspect ratios. The resolution becomes worse as the pixel volume increases, and this is due to increased detector depth rather than the overall increase in the sensitive region. For the VESPA high-resolution mode to be effective, a maximum of $1 \mathrm{~cm}$ depth is deemed acceptable. Within this limit (e.g. $0.8 \mathrm{~cm}$ ), the pixel area affects the resolution within a bracket of a few percent, with slightly better performance achieved by reducing the horizontal pitch. Moving to a more realistic configuration, the results from a cubic voxel are comparable with those obtained with cylindrical geometry ("cyl"), but there is still a deviation from the ideal infinitely thin detectors ("flat") as shown if Fig. 110. Nonetheless, according to this finding, this analysis provides enough information to identify the ideal size of the ${ }^{3} \mathrm{He}$ tubes to be used in the detector bank. Two tube sizes can be considered, as available on the market: $0.8 \mathrm{~cm}$ diameter and $1 / 2$ inch $(1.27 \mathrm{~cm})$ diameter, for both we assume a position sensitivity on their long axis equal to the diameter. From the data reported in Fig. 11, we can infer that $0.8 \mathrm{~cm}$ tubes can provide sufficient performance for high-resolution spectroscopy at high energy transfers, with the best possible resolution for ${ }^{3} \mathrm{He}$ proportional-counter detector technology. On the other hand, $1 / 2$ " tubes would provide a non-negligible detriment to resolution. This has been confirmed by additional simulations with cylindrical geometry, which are not here reported.

[Figure 11 about here.]

\section{Conclusions}

To optimise the design of large curved analysers, extensive neutron-transport simulations using analytical models and McStas have been performed. The method to design the curvature, based on the identification of a geometric locus of constant ToF from sample to detector provides a robust criterion to keep STFD at bay. It enables increased instrument performance by covering large solid angles of scattered neutrons, and provides a resolution buffer to offer further room for neutron collection via the increase in HOPG mosaicity. If one considers the maximum solid angle that can be covered with a flat-analyser layout, larger coverages up to a factor 4-4.5 can be obtained, depending on spatial and cost constraints. Concurrently, tuning of the HOPG crystal can also improve collection, nearly linearly, 
as the mosaic spread is increased. The HOPG thickness can also affect collection slightly through increased reflectivity in the energy range of interest, but gains are sub-linear when the crystals are already in the millimetre range. As a general example, taking a baseline of $0.86 \mathrm{sr}$ and $2.5^{\circ}$ mosaicity (i.e. contemporary TOSCA), a order-of-magnitude combined gain can be expected if high-mosaicity crystals become available.

Real-world effects due to manufacturing tolerances can be accounted for, and suitable limits identified. In particular, $\pm 0.5^{\circ}$ for $\mathrm{HOPG}$ mosaicity or $\pm 0.2 \mathrm{~mm}$ over multiple directions seem to provide resolution performances that are not far away from the ideal case. Concerning the detectors, $8 \mathrm{~mm}{ }^{3} \mathrm{He}$ tubes should provide the best resolution, but in principle, a thinner detector can lead to results closer to the ideal case. In principle, looser tolerances can be accepted without excessive detriment of the resolution.

Currently, a quantitative assessment and benchmarking of the mechanical properties and neutronic response of construction materials is in progress. This has the aim of providing further insights for their selection and the expected performance, with a particular attention to the background signal to be expected.

\section{Acknowledgements}

We gratefully acknowledge financial support from the VESPA Project, the STFC, the 2014-2020 CNR-STFC agreement for collaborative scientific research at ISIS. Computing resources have been provided by STFC Scientific Computing Department's SCARF cluster.

\section{References}

[1] S. Rudić, A. J. Ramirez-Cuesta, S. F. Parker, F. Fernandez-Alonso, R. S. Pinna, G. Gorini, C. G. Salzmann, S. E. McLain, N. T. Skipper, TOSCA International Beamline Review, RAL Technical Reports RAL-TR-2013-015, STFC (2013).

URL http: //purl .org/net/epubs/work/11216706

[2] S. F. Parker, F. Fernandez-Alonso, A. J. Ramirez-Cuesta, J. Tomkinson, S. Rudić, R. S. Pinna, G. Gorini, J. F. Castañon, Recent and future developments on TOSCA at ISIS, Journal of Physics: Conference Series 554 (1) (2014) 012003.

URL http: //stacks . iop.org/1742-6596/554/i=1/a=012003 
[3] R. Pinna, M. Zanetti, S. Rudić, S. Parker, J. Armstrong, S. Waller, D. Zacek, C. Smith, S. Harrison, G. Gorini, F. Fernandez-Alonso, The TOSCA Spectrometer at ISIS: the Guide Upgrade and Beyond, Journal of Physics: Conference Series 1021 (1) (2018) 012029.

URL http://stacks . iop .org/1742-6596/1021/i=1/a=012029

[4] R. S. Pinna, S. Rudić, M. J. Capstick, D. J. McPhail, D. E. Pooley, G. D. Howells, G. Gorini, F. Fernandez-Alonso, Detailed characterisation of the incident neutron beam on the TOSCA spectrometer, Nuclear Instruments and Methods in Physics Research Section A: Accelerators, Spectrometers, Detectors and Associated Equipment 870 (2017) 79 - 83. doi :https:// doi.org/10.1016/j.nima.2017.07.018.

[5] R. S. Pinna, S. Rudić, S. F. Parker, J. Armstrong, M. Zanetti, G. Škoro, S. P. Waller, D. Zacek, C. A. Smith, M. J. Capstick, D. J. McPhail, D. E. Pooley, G. D. Howells, G. Gorini, F. Fernandez-Alonso, The neutron guide upgrade of the TOSCA spectrometer, Nuclear Instruments and Methods in Physics Research Section A: Accelerators, Spectrometers, Detectors and Associated Equipment 896 (2018) 68 - 74. doi:https://doi.org/10.1016/ j.nima. 2018.04.009

[6] Accessed on 2019-07-09. [link]. URL https://neutrons.ornl.gov/vision

[7] R. S. Pinna, S. Rudić, M. Zanetti, D. Zacek, , S. F. Parker, G. Gorini, F. Fernandez-Alonso, Monte Carlo simulations for the TOSCA secondary spectrometer upgrade, RAL Technical Reports RAL-TR-2017-013, STFC (2017).

URL http: //purl.org/net/epubs/work/35959990

[8] M. Zanetti, S. Bellissima, L. del Rosso, F. Masi, M. Chowdhury, A. D. Bonis, L. D. Fresco, C. Scatigno, J. Armstrong, S. Rudić, S. Parker, M. Hartl, D. Colognesi, R. Senesi, C. Andreani, G. Gorini, F. Fernandez-Alonso, Neutronic developments on TOSCA and VESPA: Progress to date, Physica B: Condensed Matter 562 (2019) 107 - 111. doi:https://doi.org/10. $1016 / \mathrm{j}$. physb.2018.12.034.

[9] A. Fedrigo, D. Colognesi, M. Bertelsen, M. Hartl, K. Lefmann, P. P. Deen, M. Strobl, F. Grazzi, M. Zoppi, VESPA: The vibrational spectrometer for the 
European Spallation Source, Review of Scientific Instruments 87 (6) (2016) 065101. doi : 10.1063/1.4952430

[10] Mitchell, Philip C H and Parker, Stewart F and Ramirez-Cuesta, Anibal J and Tomkinson, John, Vibrational Spectroscopy with Neutrons, WORLD SCIENTIFIC, 2005. doi : 10.1142/5628.

[11] P. A. Seeger, L. L. Daemen, J. Z. Larese, Resolution of VISION, a crystalanalyzer spectrometer, Nuclear Instruments and Methods in Physics Research Section A: Accelerators, Spectrometers, Detectors and Associated Equipment 604 (3) (2009) 719 - 728. doi:https://doi .org/10.1016/ j.nima.2009.03.204.

[12] P. Willendrup, E. Farhi, K. Lefmann, McStas 1.7 - a new version of the flexible Monte Carlo neutron scattering package, Physica B: Condensed Matter 350 (1, Supplement) (2004) E735 - E737, proceedings of the Third European Conference on Neutron Scattering. doi:https://doi.org/10. $1016 / \mathrm{j}$.physb.2004.03.193.

[13] N. Takahashi, K. Shibata, T. J. Sato, Y. Kawakita, I. Tsukushi, N. Metoki, K. Nakajima, M. Arai, Study of the analyzer crystals for use in the nearbackscattering spectrometer DNA at J-PARC, Nuclear Instruments and Methods in Physics Research Section A: Accelerators, Spectrometers, Detectors and Associated Equipment 600 (1) (2009) 91 - 93. doi:https: //doi.org/10.1016/j.nima.2008.11.057. 


\section{List of Figures}

$1 \quad$ Reflection from a virtual tile centred at an arbitrary point in space. For further details see main text. . . . . . . . . . . . . . . . . 14

2 Tile position locus for VESPA analysers: (a) $E_{f}$ in meV (i.e. required orientation) on the locus. (b) Figure of merit. The yellow curve represents the optimal curvature. (c) Deviation from $t_{\text {set }}$ $(730 \mu \mathrm{s})$, in $\mu \mathrm{s}$. Positive values correspond to longer travel times. (d) Deviation from starting $E_{f}$, in meV; positive values correspond to higher energies. . . . . . . . . . . . . 15

3 (a) Relative integrated intensity on a detector bank as a function of HOPG mosaicity. (b b c) Increase of spot size on detector as a function of the HOPG mosaicity. . . . . . . . . . . . . 16

4 (a) Final-energy uncertainty as a function of HOPG mosaicity. (b) STFD as a function of HOPG mosaicity. . . . . . . . . . . . . . 17

5 Relative spectral resolution as a function of HOPG mosaicity. . . . 18

6 Tile reference system - rotations around $z$ not considered, see main text for further details. . . . . . . . . . . . . . . . . 19

$7 \quad$ Tile displacement effect at $2^{\circ}$ mosaicity, for different tolerance ranges stated as "x/y, mm; $\mathrm{z}, \mathrm{mm}$; rot, " ". (a) Average relative resolution; (b) average relative uncertainty of resolution. . . . . . 20

8 Figures of merit for the 300 random configurations, at $2^{\circ}$ mosaicity, for each tolerance range (See Fig. 7). (a) Dispersion of uncertainties with respect to ideal value. (b) Intensity fraction with respect to ideal case. . . . . . . . . . . . . . 21

9 Tile mosaic tolerances. (a) Average relative resolution for different mosaicity ranges. (b) Average relative uncertainty of resolution for different mosaicity ranges. . . . . . . . . . . . . 22

10 Figures of merit for the 300 random configurations for each mosaicity range. (a) Dispersion of uncertainties with respect to ideal csase. (b) Intensity fraction with respect to ideal case. . . . . . . . 23

11 Effect of detector voxel dimensions, at $2^{\circ}$ mosaicity. (a) Relative resolution as a function of voxel volume. (b) Relative resolution for different thickness at constant area $(0.4 \mathrm{~cm}$ square $)$ over the detector plane. (c) Comparison of resolution for constant thickness voxel with variable area and aspect ratios (Vertical vs Horizontal, vertical is along sample axis). (d) Comparison of relative resolution for flat, cylindrical and cubic voxel geometries. . . . . . . . . 24 


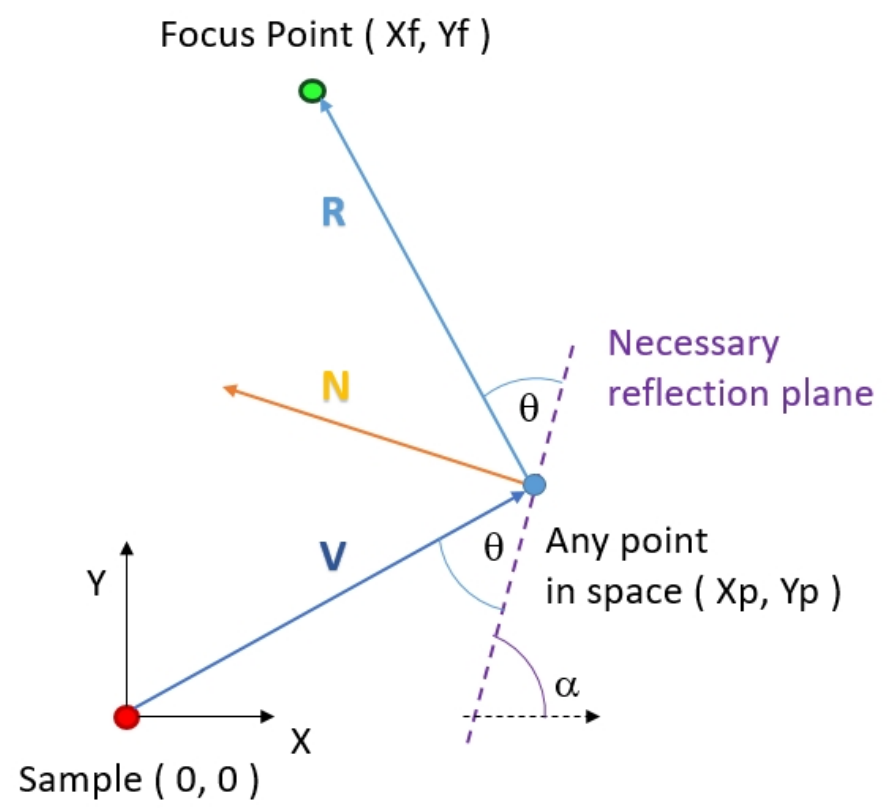

Figure 1: Reflection from a virtual tile centred at an arbitrary point in space. For further details see main text. 

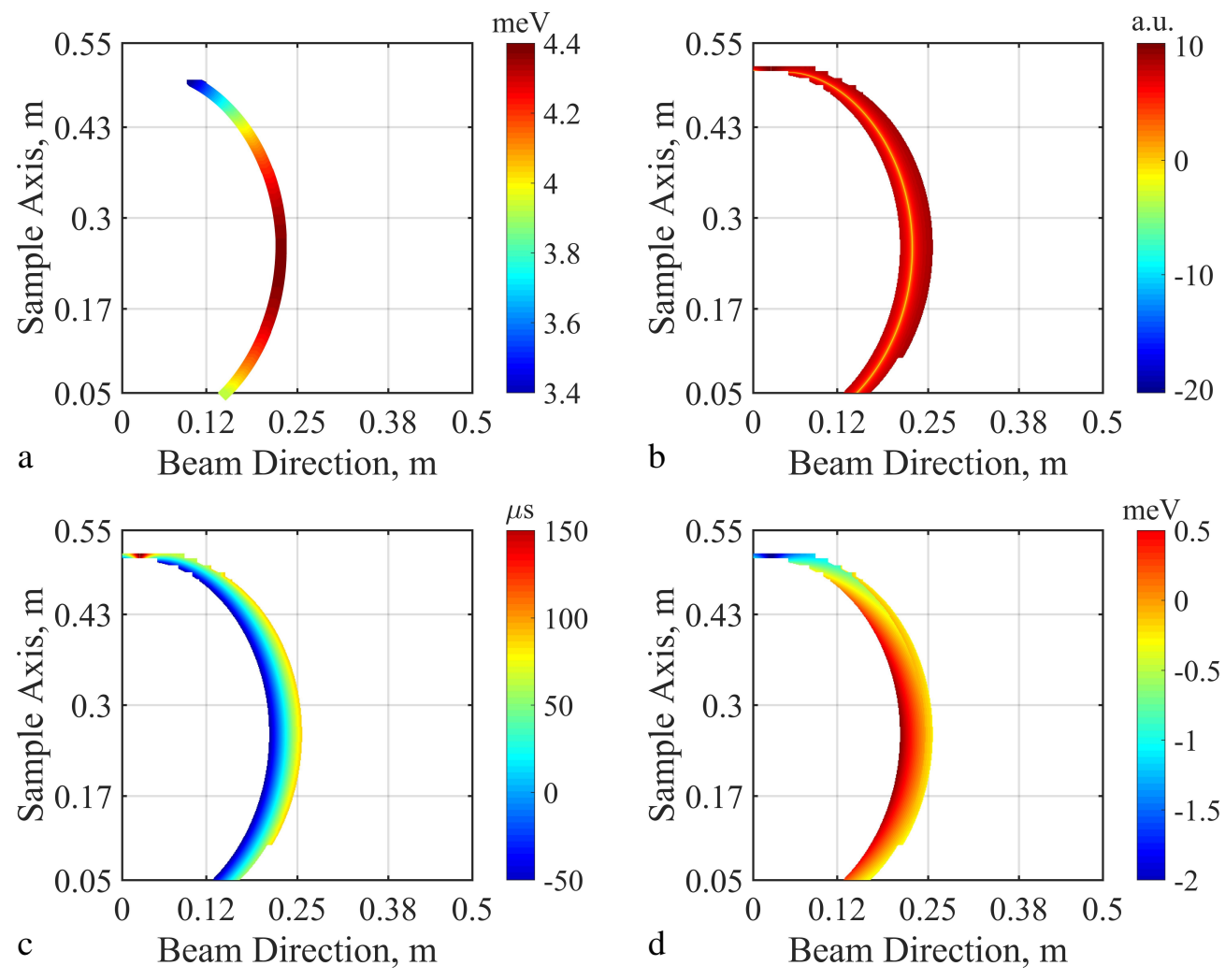

Figure 2: Tile position locus for VESPA analysers: a $E_{f}$ in $\mathrm{meV}$ (i.e. required orientation) on the locus. (b) Figure of merit. The yellow curve represents the optimal curvature. (c) Deviation from $t_{\text {set }}(730 \mu \mathrm{s})$, in $\mu \mathrm{s}$. Positive values correspond to longer travel times. (d) Deviation from starting $E_{f}$, in meV; positive values correspond to higher energies. 

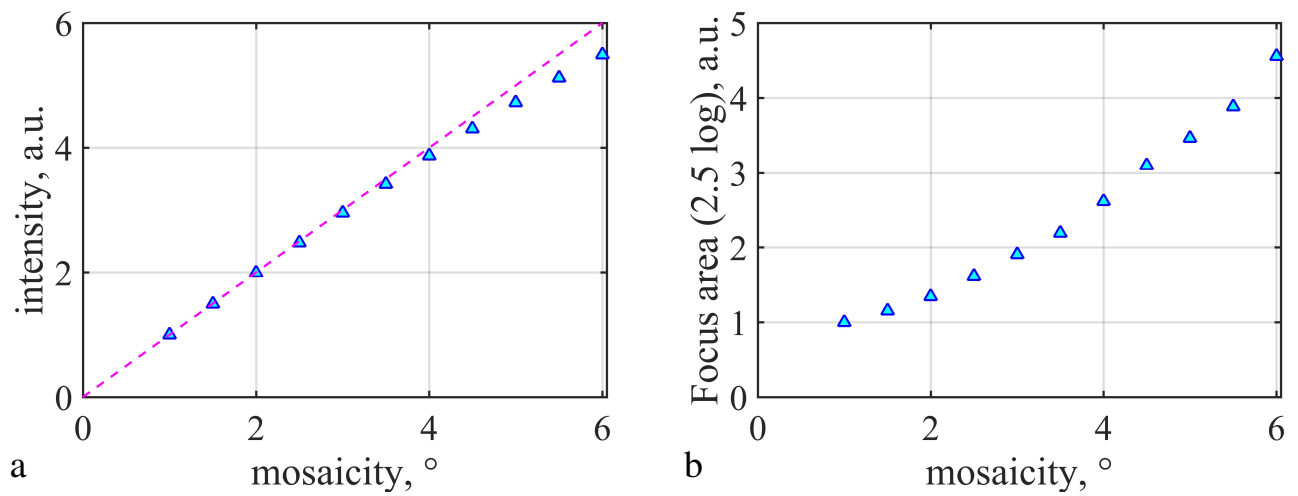

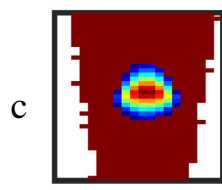

$1^{\circ}$

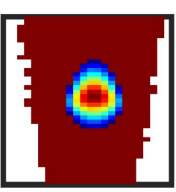

$2^{\circ}$

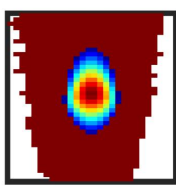

$3^{\circ}$

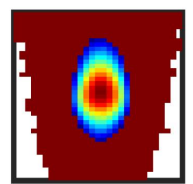

$4^{\circ}$

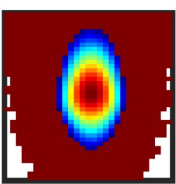

$5^{\circ}$

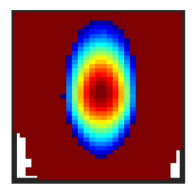

$6^{\circ}$

Figure 3: a Relative integrated intensity on a detector bank as a function of HOPG mosaicity. (bc) Increase of spot size on detector as a function of the HOPG mosaicity. 

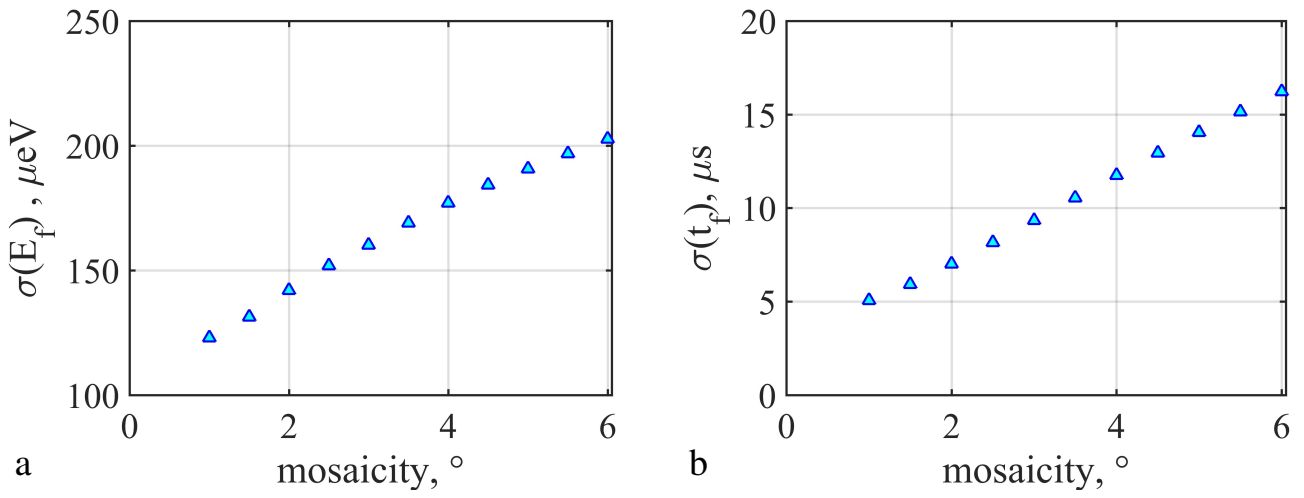

Figure 4: a Final-energy uncertainty as a function of HOPG mosaicity. (b) STFD as a function of HOPG mosaicity. 


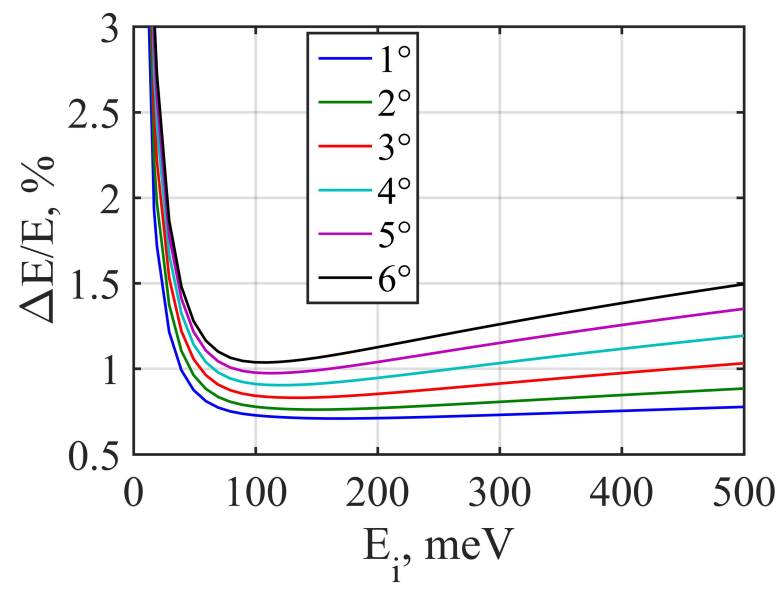

Figure 5: Relative spectral resolution as a function of HOPG mosaicity. 


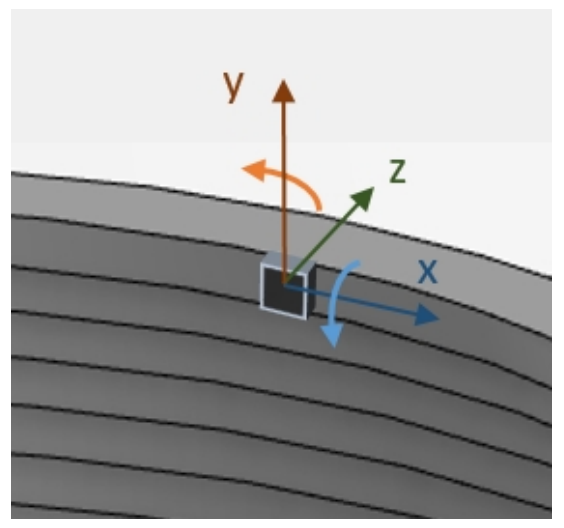

Figure 6: Tile reference system - rotations around $z$ not considered, see main text for further details. 

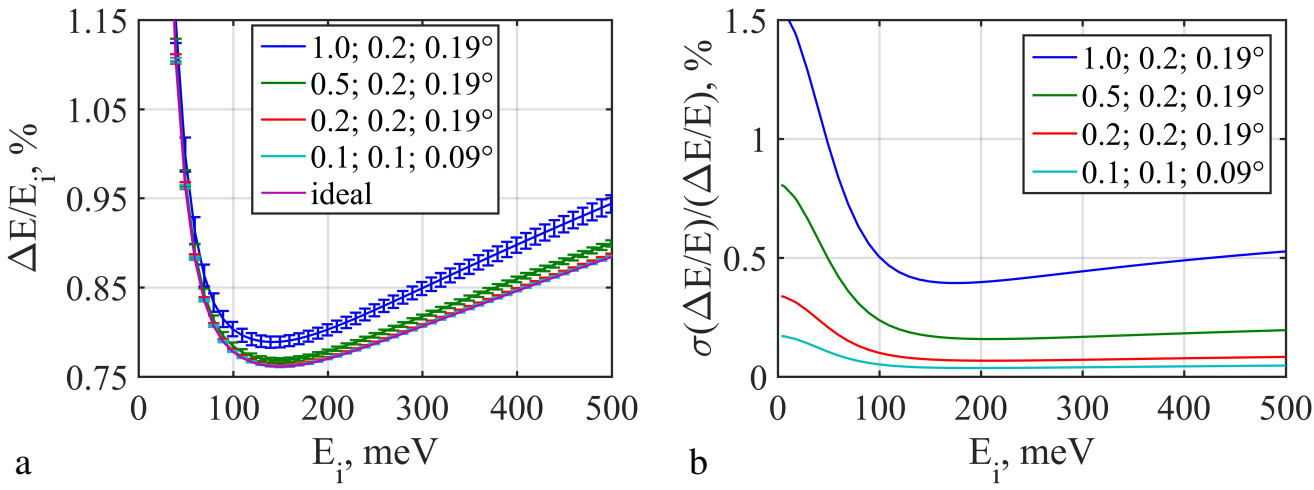

Figure 7: Tile displacement effect at $2^{\circ}$ mosaicity, for different tolerance ranges stated as " $\mathrm{x} / \mathrm{y}$, $\mathrm{mm} ; \mathrm{z}, \mathrm{mm}$; rot, ".. (a) Average relative resolution ; (b) average relative uncertainty of resolution. 

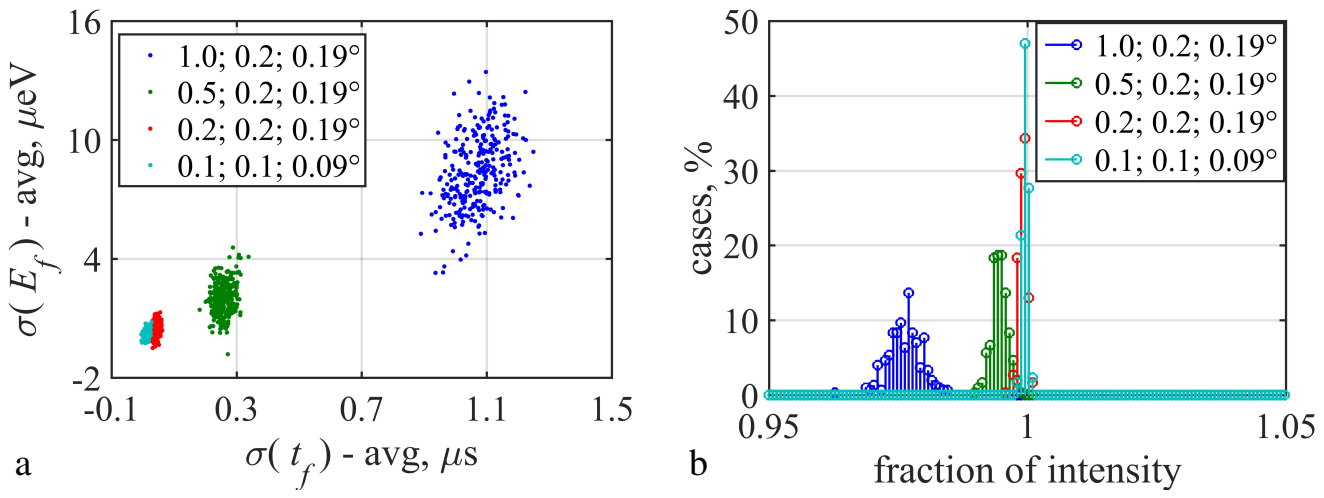

Figure 8: Figures of merit for the 300 random configurations, at $2^{\circ}$ mosaicity, for each tolerance range (See Fig. 7). (a) Dispersion of uncertainties with respect to ideal value. (b) Intensity fraction with respect to ideal case. 

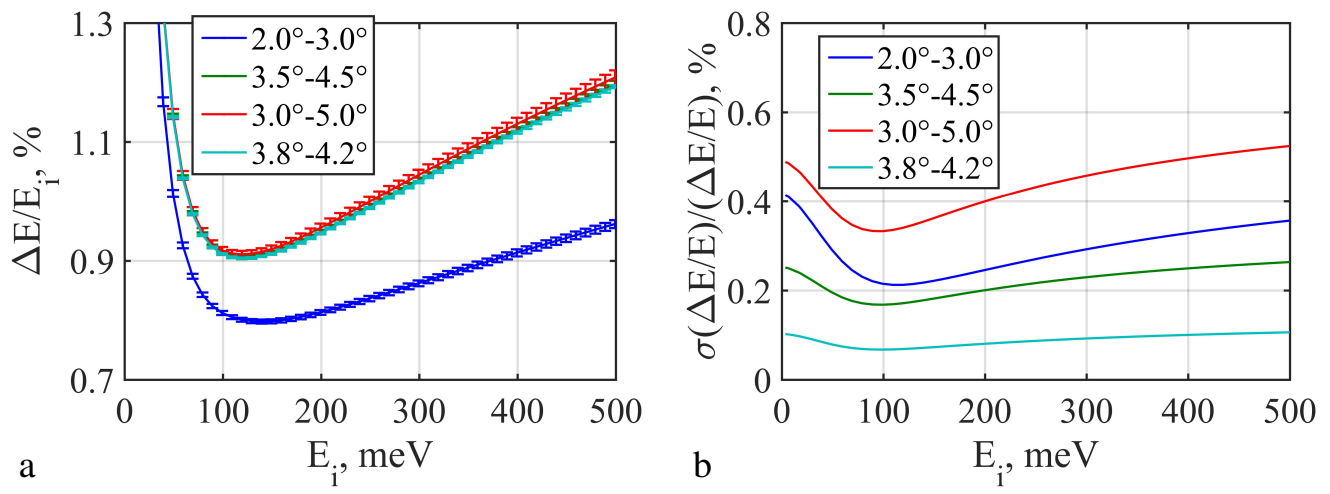

Figure 9: Tile mosaic tolerances. (a) Average relative resolution for different mosaicity ranges. (b) Average relative uncertainty of resolution for different mosaicity ranges. 

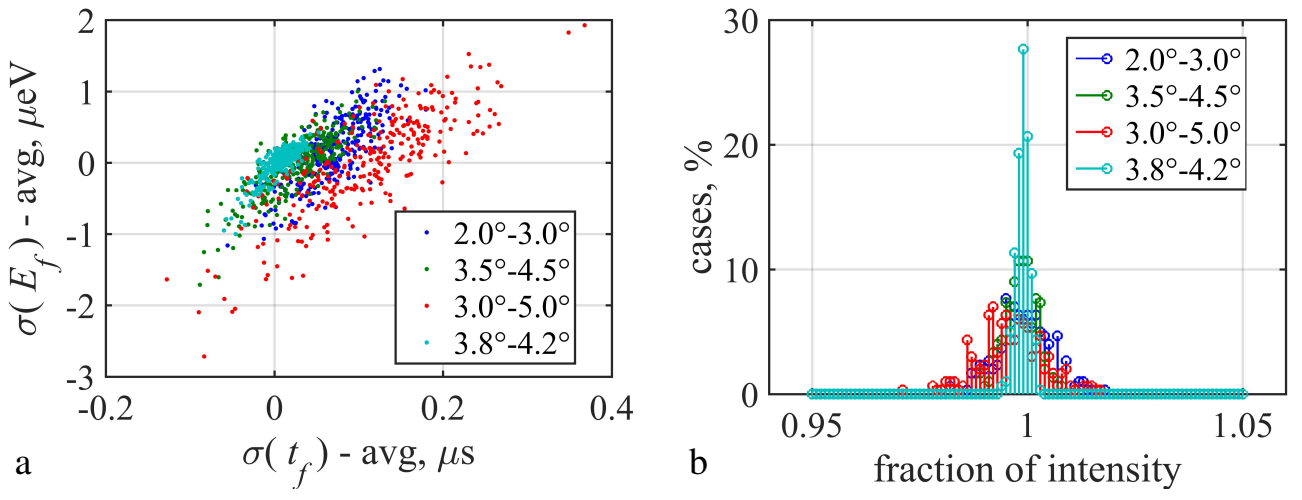

Figure 10: Figures of merit for the 300 random configurations for each mosaicity range. (a) Dispersion of uncertainties with respect to ideal csase. (b) Intensity fraction with respect to ideal case. 

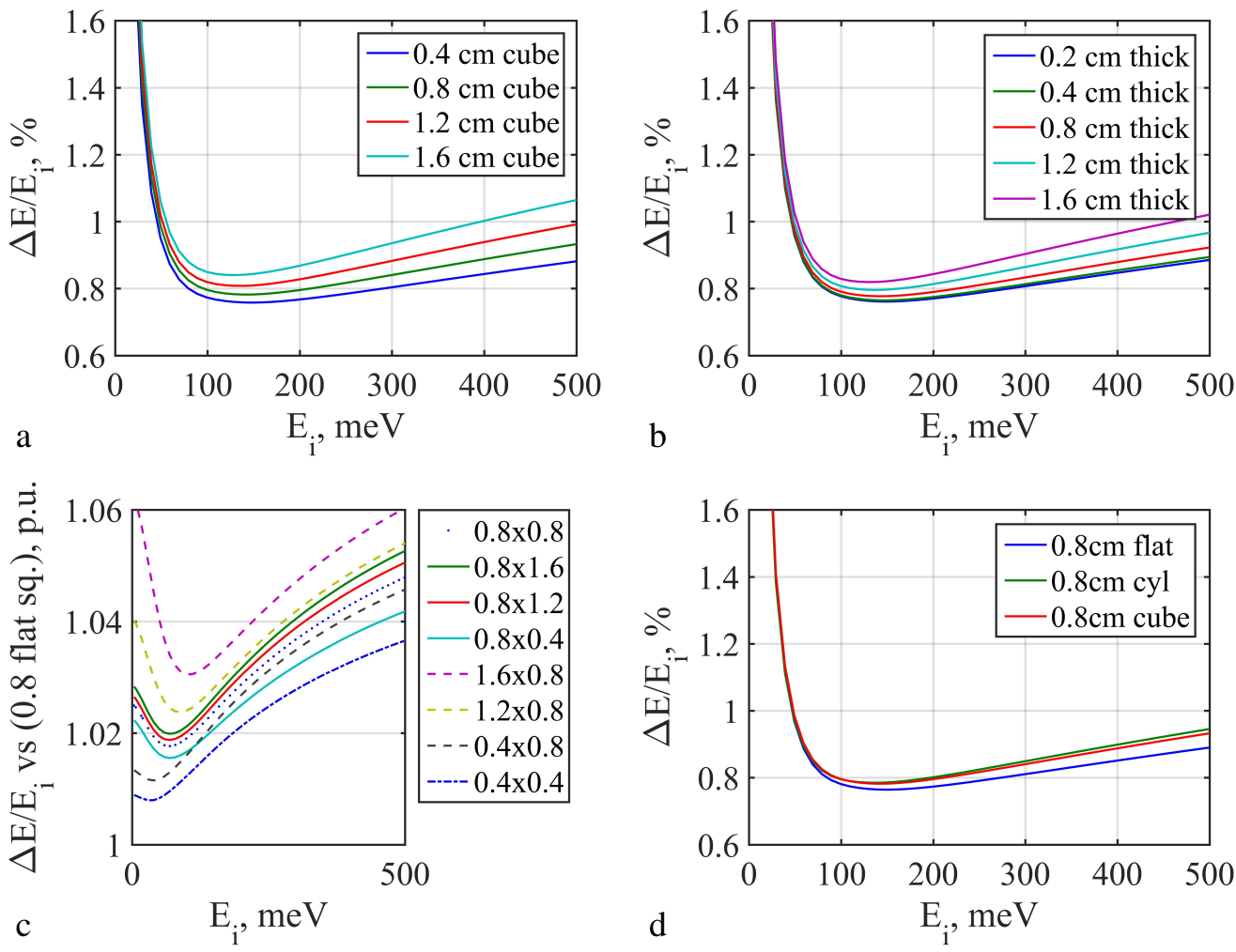

Figure 11: Effect of detector voxel dimensions, at $2^{\circ}$ mosaicity. (a) Relative resolution as a function of voxel volume. (b) Relative resolution for different thickness at constant area $(0.4$ $\mathrm{cm}$ square) over the detector plane. (c) Comparison of resolution for constant thickness voxel with variable area and aspect ratios (Vertical vs Horizontal, vertical is along sample axis). (d) Comparison of relative resolution for flat, cylindrical and cubic voxel geometries. 